Sādhanā Vol. 37, Part 6, December 2012, pp. 665-674. (C) Indian Academy of Sciences

\title{
Solving the minimum flow problem with interval bounds and flows
}

\author{
MEHDI GHIYASVAND \\ Department of Mathematics, Faculty of Science, Bu-Ali Sina University, \\ Hamedan 65178-38695, Iran \\ e-mail: meghiyasvand@yahoo.com
}

MS received 30 October 2011; revised 14 June 2012; accepted 3 July 2012

\begin{abstract}
The minimum cost flow problem with interval data can be solved using two minimum cost flow problems with crisp data. In this paper, the idea of Ghiyasvand was extended for solving the minimum flow problem with interval-valued lower, upper bounds and flows. This problem can be solved using two minimum flow problems with crisp data. Then, this result is extended to networks with fuzzy lower, upper bounds and flows.
\end{abstract}

Keywords. Network flows; minimum flow problem; interval data.

\section{Introduction}

Let $D=(N, A)$ be a network by a set $N$ of $n$ nodes and a set $A$ of $m$ directed arcs. We also associate with each arc $e=(i, j) \in A$ an upper bound $u(e)$ that denotes the maximum amount that can flow on the arc and a lower bound $l(e)$ that denotes the minimum amount that must flow on the arc. To define the minimum flow problem, we distinguish two special nodes in the network $D$ : a source node $s$ and a sink node $t$. The problem is to find the minimum flow from the source node $s$ to the sink node $t$ that satisfy the lower and upper bounds and balance constraints at all nodes. The decision variables in the minimum cost flow problem are arc flows and we represent the flow on an arc $e=(i, j) \in A$ by $f(e)$ or $f(i, j)$. The minimum flow problem can state formally as follows.

The minimum flow problem is one of network flows that computes the minimum flow between two given nodes, called source and sink nodes. There are several approaches to solve the 
minimum flow problem: (i) decreasing path algorithms (Ciupala \& Ciurea 2001a, b, 2004), (ii) preflow algorithms (Ciupala 2006; Ciurea 2008; Ciupala \& Ciurea 2001a, 2003, 2007, 2008), (iii) minimax algorithm which consists of finding a maximum flow from the sink node to the source node in the residual network (Bang-Jenson \& Gutin 2001; Ciupala \& Ciurea 2001b), (iv) using dynamic tree implementations (Ciurea et al 2008a). Also, Ciurea \& Deaconu (2009) and Ciurea et al (2008b) solved the minimum flow problem for bipartite networks, and Ciurea \& Deaconu (2007) solved the inverse minimum flow problem.

In Ghiyasvand (2011), a new method to solve the minimum cost flow problem with interval data is presented. First, it solves a minimum cost flow problem with lower bounds, flows, and costs, also a minimum cost flow problem with upper bounds, flows, and costs. Then, the method combines two solutions as an interval solution. Ghiyasvand (2011) proved that the interval solution is optimal for the minimum cost flow problem with interval bounds, flows, and costs. In this paper, this idea is extended to solve the minimum flow problem with interval bounds and flows. We show that the minimum flow problem can be solved using two minimum flow problems with crisp data.

This paper consists of five sections including Introduction. Section 2 presents notation and reviews of some results about crisp, interval and fuzzy data which are used in the subsequent sections. In section 3 , the relationship between the minimum flow problems with interval data and crisp data is shown. The minimum flow problem with fuzzy data is described in section 4 . Finally, conclusions are provided in section 5.

\section{Preliminaries}

\subsection{Interval arithmetics}

Let $I$ denote the class of non-empty compact intervals $[\underline{x}, \bar{x}]$ on $[0, \infty)$. If $\underline{x}=\bar{x}=a$, identify the interval with the real number $a$.

Definition 1 (Moore 1978). Let $\left[\underline{x}_{1}, \bar{x}_{1}\right]$ and $\left[\underline{x}_{2}, \bar{x}_{2}\right]$ be two compact intervals, then

$$
\begin{gathered}
{\left[\underline{x}_{1}, \bar{x}_{1}\right] \leq\left[\underline{x}_{2}, \bar{x}_{2}\right] \text { if } \underline{x}_{1} \leq \underline{x}_{2} \text { and } \bar{x}_{1} \leq \bar{x}_{2},} \\
{\left[\underline{x}_{1}, \bar{x}_{1}\right]+\left[\underline{x}_{2}, \bar{x}_{2}\right]=\left[\underline{x}_{1}+\underline{x}_{2}, \bar{x}_{1}+\bar{x}_{2}\right],} \\
{\left[\underline{x}_{1}, \bar{x}_{1}\right]\left[\underline{x}_{2}, \bar{x}_{2}\right]=\left[\min \left(\underline{x}_{1} \underline{x}_{2}, \underline{x}_{1} \bar{x}_{2}, \bar{x}_{1} \underline{x}_{2}, \bar{x}_{1} \bar{x}_{2}\right), \max \left(\underline{x}_{1} \underline{x}_{2}, \underline{x}_{1} \bar{x}_{2}, \bar{x}_{1} \underline{x}_{2}, \bar{x}_{1} \bar{x}_{2}\right)\right] .}
\end{gathered}
$$

The infimum and supremum of $\left[\underline{x}_{1}, \bar{x}_{1}\right]$ and $\left[\underline{x}_{2}, \bar{x}_{2}\right]$, respectively, are defined by

$$
\begin{aligned}
& {\left[\underline{x}_{1}, \bar{x}_{1}\right] \wedge\left[\underline{x}_{2}, \bar{x}_{2}\right]=\left[\min \left\{\underline{x}_{1}, \underline{x}_{2}\right\}, \min \left\{\bar{x}_{1}, \bar{x}_{2}\right\}\right],} \\
& {\left[\underline{x}_{1}, \bar{x}_{1}\right] \vee\left[\underline{x}_{2}, \bar{x}_{2}\right]=\left[\max \left\{\underline{x}_{1}, \underline{x}_{2}\right\}, \max \left\{\bar{x}_{1}, \bar{x}_{2}\right\}\right] .}
\end{aligned}
$$

If $\left[\underline{x}_{1}, \bar{x}_{1}\right], \ldots,\left[\underline{x}_{n}, \bar{x}_{n}\right] \in I$, then the infimum $\wedge_{i}\left[\underline{x}_{i}, \bar{x}_{i}\right]$ and supremum $\vee_{i}\left[\underline{x}_{i}, \bar{x}_{i}\right]$ are welldefined and

$$
\sum_{\{i \mid}\left[\underline{x}_{i}, \bar{x}_{i}\right]=\left[\sum_{\{i \mid, ., n\}} \underline{x}_{i}, \sum_{\{i \mid 1, . ., n\}} \bar{x}_{i}\right] .
$$




\subsection{Zadeh's extension principle}

Let $X$ be a non-empty set, for example $X=R^{n}$. A fuzzy set $A \subset X$ is characterized by the membership function $\mu_{A}: X \rightarrow[0,1]$, where $\mu_{A}(x)$ is the degree of membership of $x$ in set $A$. The $\beta$-level set of a fuzzy set $A$, denoted by $[A]^{\beta}$, is the crisp subset of $X$ that contains all of elements with at least the given degree of membership $\beta$ :

$$
[A]^{\beta}=\left\{x \in X \mid \mu_{A}(x) \geq \beta\right\} .
$$

Fuzzy numbers are the class $\xi^{1}$ of normal, upper semicontinuous fuzzy convex fuzzy sets on $R$. That is, $A$ is a fuzzy number if all the level sets $[A]^{\beta}, 0 \leq \beta \leq 1$, are compact intervals and there is at least one $z \in R$ such that $\mu_{A}(z)=1$.

Zadeh's extension principle (Hanss 2005) is said to be one of the most important tools in fuzzy logic. It gives means to generalize non-fuzzy concepts, e.g., mathematical operations, to fuzzy sets. Let $A_{1}, A_{2}, \ldots, A_{n}$ be fuzzy sets, defined on $X_{1}, X_{2}, \ldots, X_{n}$, and $f$ be a function $f: X_{1} \times X_{2} \times \ldots \times X_{n} \rightarrow V$. Zadeh's extension principle of $f$ operating on $A_{1}, A_{2}, \ldots, A_{n}$ gives a membership function (fuzzy set $F$ )

$$
\mu_{F}(v)=\sup _{\left\{u_{1}, . ., u_{n} \in f^{-1}(v)\right\}} \min \left(\mu_{A_{1}}\left(u_{1}\right), . ., \mu_{A_{n}}\left(u_{n}\right)\right.
$$

where the inverse of $f$ exists. Otherwise define $\mu_{F}(v)=0$. Function $f$ is called inducing mapping. If the domain is either discrete or compact, sup-min can be replaced by $\max -\min$.

Property 1. Basically, Zadeh's extension principle says that a fuzzy set is a collection of intervals with a membership associated ( $\beta$-levels) to them (Nguyen 1978; Nguyen \& Kreinovich 1996). Thus, whenever a result can mention about the intervals, it can mention about the fuzzy sets.

Zadeh's extension principle is equivalent to the partition into $\beta$-cuts, using addition and scalar multiplication of convex sets, when the fuzzification uses Definition 1 for max-min and order operations.

Conclusion 1 (Diamond 2001). If a network flow theorem can be proved for compact intervalvalued flows, capacities and costs, using Definition 1 for max, min and order properties, then a similar theorem will follow for fuzzy network quantities, which characterizes a fuzzy number by its level sets, which are compact intervals.

\subsection{The machine set-up problem}

The famous application for the minimum flow problem is the machine set-up problem that is presented in this part: A job shop needs to perform $\mathrm{p}$ tasks on a particular day. The start and end times of each performance is given, the workers must perform these tasks according to the schedule so that exactly one worker performs each task. A worker can not work on two jobs at the same time. Also we have the set-up time required for a worker to go from one task to another. We wish to find the minimum number of workers to perform the tasks, This problem can be formulated as a minimum flow problem (Ciupala \& Ciurea 2001a). 


\section{The minimum flow problem with interval data}

Consider a network $D=(N, A)$ such that the lower bounds, upper bounds, and flows of each arc are known only to fall within specific ranges expressed as compact intervals $\bar{l}, \bar{u}$, and $\bar{f}$, respectively. Thus, for each arc e, we have

$$
\begin{aligned}
l(e) \in \bar{l}(e) & =\left[l_{l}(e), r_{l}(e)\right], \\
u(e) \in \bar{u}(e) & =\left[l_{u}(e), r_{u}(e)\right], \text { and } \\
f(e) \in \bar{f}(e) & =\left[l_{f}(e), r_{f}(e)\right],
\end{aligned}
$$

where $l_{l}(e), r_{l}(e), l_{u}(e), r_{u}(e), l_{f}(e)$ and $r_{f}(e)$ are non-negative real values. The minimum flow problem with compact interval-valued lower and upper bounds, and flows is:

$$
\begin{gathered}
\min \left[v_{l}, v_{r}\right] \\
\text { s.t. } \sum_{\{y \mid(x, y) \in A\}} \bar{f}(x, y)-\sum_{\{y \mid(y, x) \in A\}} \bar{f}(y, x)= \begin{cases}{\left[v_{l}, v_{r}\right]} & \text { for } x=s, \\
{[0,0]} & \text { for all } x \in N-\{s, t\}, \\
-\left[v_{l}, v_{r}\right] & \text { for } x=t, \\
\bar{l}(e) \leq \bar{f}(e) \leq \bar{u}(e), & \forall e \in A .\end{cases}
\end{gathered}
$$

We call this problem the interval-min flow problem. Let $\bar{f}^{*}$ be an answer of this problem. For each arc $e$, we define any element of the interval $\bar{f}^{*}(e)$ as an answer for the interval-min flow problem. By Definition 1, Conditions (4) and (5) can be written by

$$
\begin{gathered}
\left.\left[\sum_{\{y \mid(x, y) \in A\}} l_{f}(x, y), \sum_{\{y \mid(x, y) \in A\}} r_{f}(x, y)\right\}\right]-\left[\sum_{\{y \mid(y, x) \in A\}} l_{f}(y, x), \sum_{\{y \mid(y, x) \in A\}} r_{f}(y, x)\right] \\
=\left\{\begin{array}{cc}
{\left[v_{l}, v_{r}\right] \quad \text { for } x=s,} & \\
{[0,0] \quad \text { for all } x \in N-\{s, t\},} & \\
-\left[v_{l}, v_{r}\right] \quad \text { for } x=t, & \forall e \in A, \\
l_{l}(e) \leq l_{f}(e) \leq l_{u}(e), & \forall e \in A .
\end{array}\right.
\end{gathered}
$$

Therefore, a flow $\bar{f}$ is feasible for the interval-min flow problem if it satisfies in (6), (7) and (8). Thus the interval-min flow problem can be written as follows

$$
\bigwedge\left[v_{l}, v_{r}\right]: f \text { satisfies in (6), (7), and (8). }
$$

We define the l-min flow problem by the following:

$$
\begin{gathered}
\min v_{l} \\
\text { s.t. } \sum_{\{y \mid(x, y) \in A\}} l_{f}(x, y)-\sum_{\{y \mid(y, x) \in A\}} l_{f}(y, x)=\left\{\begin{array}{cl}
v_{l} & \text { for } x=s, \\
0 & \text { for all } x \in N-\{s, t\}, \\
-v_{l} & \text { for } x=t, \\
l_{l}(e) \leq l_{f}(e) \leq l_{u}(e), & \forall e \in A .
\end{array}\right.
\end{gathered}
$$


We also define the r-min flow problem by the following:

$$
\begin{gathered}
\text { min } v_{r} \\
\text { s.t. } \sum_{\{y \mid(x, y) \in A\}} r_{f}(x, y)-\sum_{\{y \mid(y, x) \in A\}} r_{f}(y, x)=\left\{\begin{array}{cl}
v_{r} & \text { for } x=s, \\
0 & \text { for all } x \in N-\{s, t\}, \\
-v_{r} & \text { for } x=t,
\end{array}\right. \\
r_{l}(e) \leq r_{f}(e) \leq r_{u}(e),
\end{gathered}
$$

The relationship among the $l$-min flow, $r$-min flow and interval-min flow problems is shown by the next theorem.

Theorem 1. Supposing that $l_{f_{1}^{*}}$ (resp. $r_{f_{2}^{*}}$ ) is an optimal flow for the l-min (resp. r-min) flow problem. Then $\bar{f}^{*}=\left[l_{f_{1}^{*}}, r_{f_{2}^{*}}\right]$ is an optimal flow for the interval-min flow problem.

Proof. We first show the flow $\bar{f}^{*}$ is a feasible flow for the interval-min flow problem. By the feasibility of $l_{f_{1}^{*}}$ in the $l$-min flow problem, we get

$$
\begin{array}{cc}
l_{l}(e) \leq l_{f_{1}^{*}}(e) \leq l_{u}(e), & \forall e \in A, \\
\sum_{\{y \mid(x, y) \in A\}} l_{f_{1}^{*}}(x, y)-\sum_{\{y \mid(y, x) \in A\}} l_{f_{1}^{*}}(y, x)=0, & \forall x \in N-\{s, t\} .
\end{array}
$$

In a same way, $r_{f_{2}^{*}}$ is a feasible flow for the $l$-min flow problem, so we have

$$
\begin{array}{cc}
r_{l}(e) \leq r_{f_{2}^{*}}(e) \leq r_{u}(e), & \forall e \in A, \\
\sum_{\{y \mid(x, y) \in A\}} r_{f_{2}^{*}}(x, y)-\sum_{\{y \mid(y, x) \in A\}} r_{f_{2}^{*}}(y, x)=0, & \forall x \in N-\{s, t\} .
\end{array}
$$

By (6), (11), and (13), $\bar{f}^{*}$ satisfies in (4) and, by (7), (8), (10) and (12), it satisfies in (5). Thus, $\bar{f}^{*}$ is a feasible flow for the interval-min flow problem. The flow $v_{1}^{*}$ (resp. $v_{r}^{*}$ ) is optimal for the $l$-min flow problem (resp. the $r$-min flow problem), so, by (9) and Definition 1 , we yield that $\left[v_{1}^{*}, v_{r}^{*}\right]$ is an optimal flow for the interval-min flow problem.

Therefore, by Theorem 1, for solving the interval-min flow problem, it is enough that we solve the $l$-min and $r$-min flow problems, which yields the following theorem.

Theorem 2. The minimum flow problem with interval-valued lower bounds, upper bounds, and flows is solved using two minimum flow problems with crisp data.

\section{The minimum flow problem according with Zadeh's extension principle}

In this section, the minimum flow problem with fuzzy lower and upper bounds, and flows is solved using Theorem 2. Consider a network $D=(N, A)$ with fuzzy lower and upper bounds, and flows $\tilde{l}, \tilde{u}$, and $\tilde{f}$, respectively. We call the minimum cost flow problem with fuzzy data as the fuzzy-min flow problem. As it was mentioned is section 2.3, the interval representation of the $\beta$-levels allows to extend classical interval arithmetic to the case of fuzzy numbers. Interval 
arithmetic can be directly applied to every $\beta$-level to obtain the resulting fuzzy set. For each $0 \leq \beta \leq 1$ and each arc $e \in A$, we define the $\beta$-level sets corresponding to $\tilde{l}, \tilde{u}$, and $\tilde{f}$ as follows

$$
\begin{gathered}
{[\tilde{l}(e)]^{\beta}=\bar{l}(e, \beta)=\left[l_{l}(e, \beta), r_{l}(e, \beta)\right],} \\
{[\tilde{u}(e)]^{\beta}=\bar{u}(e, \beta)=\left[l_{u}(e, \beta), r_{u}(e, \beta)\right], \text { and }} \\
{[\tilde{f}(e)]^{\beta}=\bar{f}(e, \beta)=\left[l_{f}(e, \beta), r_{f}(e, \beta)\right] .}
\end{gathered}
$$

The minimum flow problem with compact interval-valued lower and upper bounds, and flows

$$
\begin{aligned}
& \min \left[v_{l}(\beta), v_{r}(\beta)\right] \\
& {\left[\sum_{\{y \mid(x, y) \in A\}} l_{f}((x, y), \beta), \sum_{\{y \mid(x, y) \in A\}} r_{f}((x, y), \beta)\right]} \\
& -\left[\sum_{\{y \mid(y, x) \in A\}} l_{f}((y, x), \beta), \sum_{\{y \mid(y, x) \in A\}} r_{f}((y, x), \beta)\right] \\
& = \begin{cases}{\left[v_{l}(\beta), v_{r}(\beta)\right]} & \text { for } i=s, \\
{[0,0]} & \text { for all } i \in N-\{s, t\}, \\
-\left[v_{l}(\beta), v_{r}(\beta)\right] & \text { for } i=t,\end{cases} \\
& l_{l}(e, \beta) \leq l_{f}(e, \beta) \leq l_{u}(e, \beta), \quad \forall e \in A, \\
& r_{l}(e, \beta) \leq r_{f}(e, \beta) \leq r_{u}(e, \beta), \quad \forall e \in A .
\end{aligned}
$$

We call the interval-valued network with data (14), (15) and (16) as the $\beta$-interval min flow network. The interval flow $[\tilde{f}]^{\beta}$ is feasible in the $(D, \beta)$ network if it satisfies in (18), (19), and (20). Therefore $\tilde{f}$ is a feasible flow for the fuzzy-min flow problem if, at each $\beta$-level, $[\tilde{f}]^{\beta}$ is a feasible flow in the the $\beta$-interval min flow problem. At each $\beta$-level, we define the $\beta$-interval min flow problem by the following:

$$
\begin{array}{ll}
\min & {\left[v_{l}(\beta), v_{r}(\beta)\right]} \\
\text { s.t. } & \bar{f}(., \beta) \text { satisfies in }(18),(19), \text { and }(20) .
\end{array}
$$

Hence, for each $0 \leq \beta \leq 1$, an interval-valued minimum flow $\bar{f}^{*}(e, \beta)=\left[l_{f}(e, \beta)\right.$, $\left.r_{f^{*}}(e, \beta)\right]$, for each arc $e$, is found by solving the the $\beta$-interval min flow problem. By 
Theorem $3, l_{f *}(e, \beta)$ 's and $r_{f^{*}}(e, \beta)$ 's are computed using two $l-\beta$-interval and $r-\beta$-interval min flow problems defined as follows

The $l-\beta$-interval min flow problem:

$$
\begin{gathered}
\min v_{l}(\beta) \\
\text { s.t. } \sum_{\{y \mid(x, y) \in A\}} l_{f}((x, y), \beta)-\sum_{\{y \mid(y, x) \in A\}} l_{f}((y, x), \beta)= \begin{cases}v_{l}(\beta) & \text { for } x=s, \\
0 & \text { for all } x \in N-\{s, t\}, \\
-v_{l}(\beta) & \text { for } x=t, \\
l_{l}(e, \beta) \leq l_{f}(e, \beta) \leq l_{u}(e, \beta), & \forall e \in A .\end{cases}
\end{gathered}
$$

$r-\beta$-interval min flow problem:

$$
\begin{array}{cl}
\min v_{r}(\beta) \\
\text { s.t. } \sum_{\{y \mid(x, y) \in A\}} r_{f}((x, y), \beta)-\sum_{\{y \mid(y, x) \in A\}} r_{f}((y, x), \beta)= \begin{cases}v_{r}(\beta) & \text { for } x=s, \\
0 & \text { for all } x \in N-\{s, t\}, \\
-v_{r}(\beta) & \text { for } x=t, \\
r_{l}(e, \beta) \leq r_{f}(e, \beta) \leq r_{u}(e, \beta), & \forall e \in A .\end{cases}
\end{array}
$$

Since the interval representation of the $\beta$-levels are used instead of the fuzzy numbers, by Property 1 , the result is accorded with Zadeh's extension principle. In general, any function of $k$ intervals $F\left(A_{1}, A_{2}, . ., A_{k}\right)$ of $k$ intervals $A_{1}, A_{2}, . ., A_{k}$ can be extended to fuzzy by defining $\left[F\left(A_{1}, A_{2}, . ., A_{k}\right)\right]^{\beta}=F\left(\left[A_{1}\right]^{\beta},\left[A_{2}\right]^{\beta}, . .,\left[A_{K}\right]^{\beta}\right)$.

However, unless $\mathrm{F}$ preserves inclusion, in order to get a fuzzy number as the result, we must modify the definition so that the level set $\beta$ is a subset of $\beta^{\prime}$ if $\beta>\beta^{\prime}$. Therefore we define (Bondia et al 2006):

$$
\left[F\left(A_{1}, A_{2}, . ., A_{k}\right)\right]^{\beta}=\bigcap_{0 \leq \beta^{\prime} \leq \beta} F\left(\left[A_{1}\right]^{\beta^{\prime}},\left[A_{2}\right]^{\beta^{\prime}}, . .,\left[A_{K}\right]^{\beta^{\prime}}\right) .
$$

For a $0 \leq \beta \leq 1$ and its $(D, \beta)$ problem, consider an interval-valued minimum flow $\bar{f}^{*}(e, \beta)=$ $\left[l_{f^{*}}(e, \beta), r_{f^{*}}(e, \beta)\right]$, for each arc $e$. Let $z^{*}(\beta)=\sum_{e \in A} \bar{c}(e, \beta) \bar{f}^{*}(e, \beta)$.

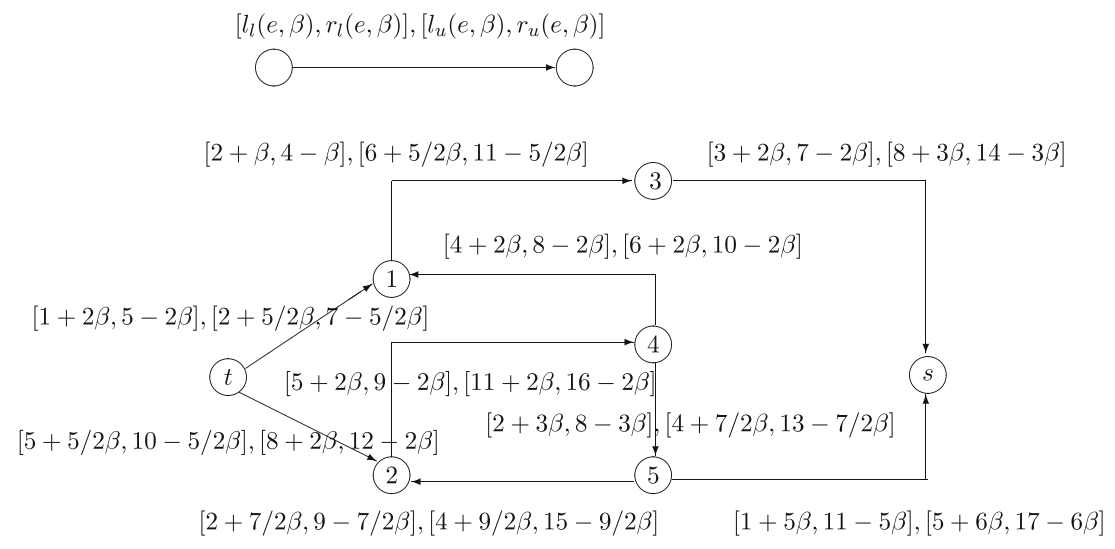

Figure 1. An example network with fuzzy bounds and flows. 


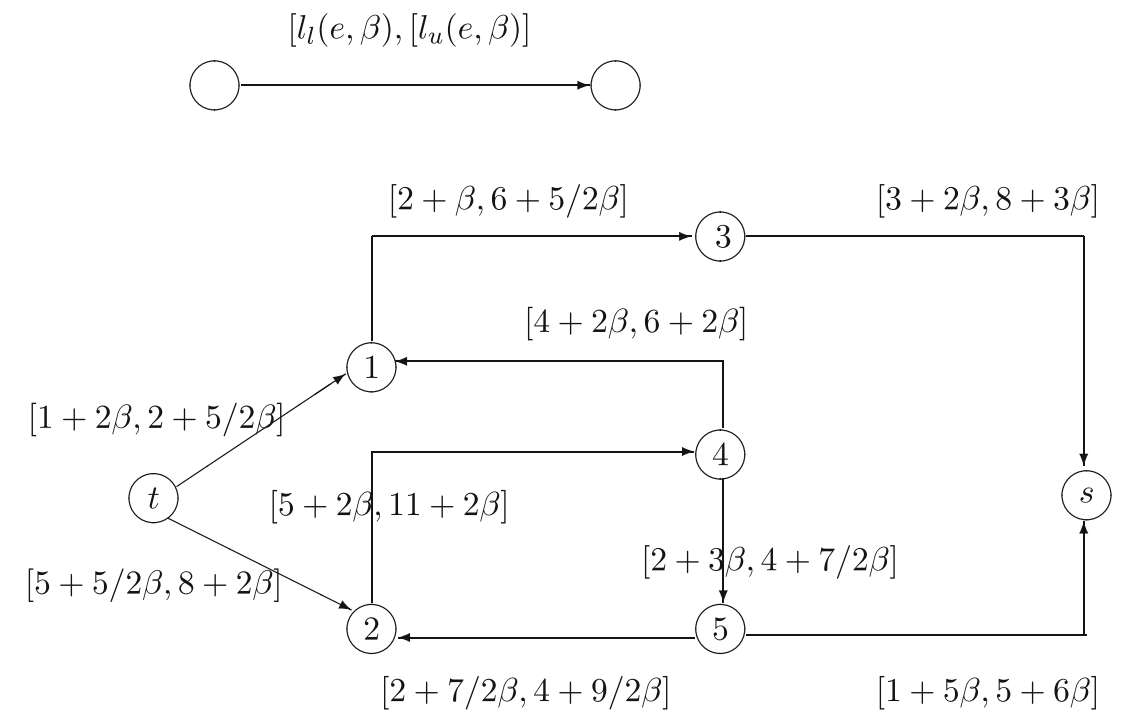

Figure 2. The network corresponding to the $l-\beta$-interval min flow problem.

The intervals $z^{*}(\beta)$ 's may not define a fuzzy set, because they might not be nested. Therefore, the following definition is used so that we have nested level sets (i.e., level set $\beta$ is a subset of $\beta^{\prime}$ if $\left.\beta>\beta^{\prime}\right)$ :

$$
\left[z^{*}\right]^{\beta}=\bigcap_{0 \leq \beta^{\prime} \leq \beta} z^{*}\left(\beta^{\prime}\right) .
$$

The level sets $\left[z^{*}\right]^{\beta}$ 's define a fuzzy number.

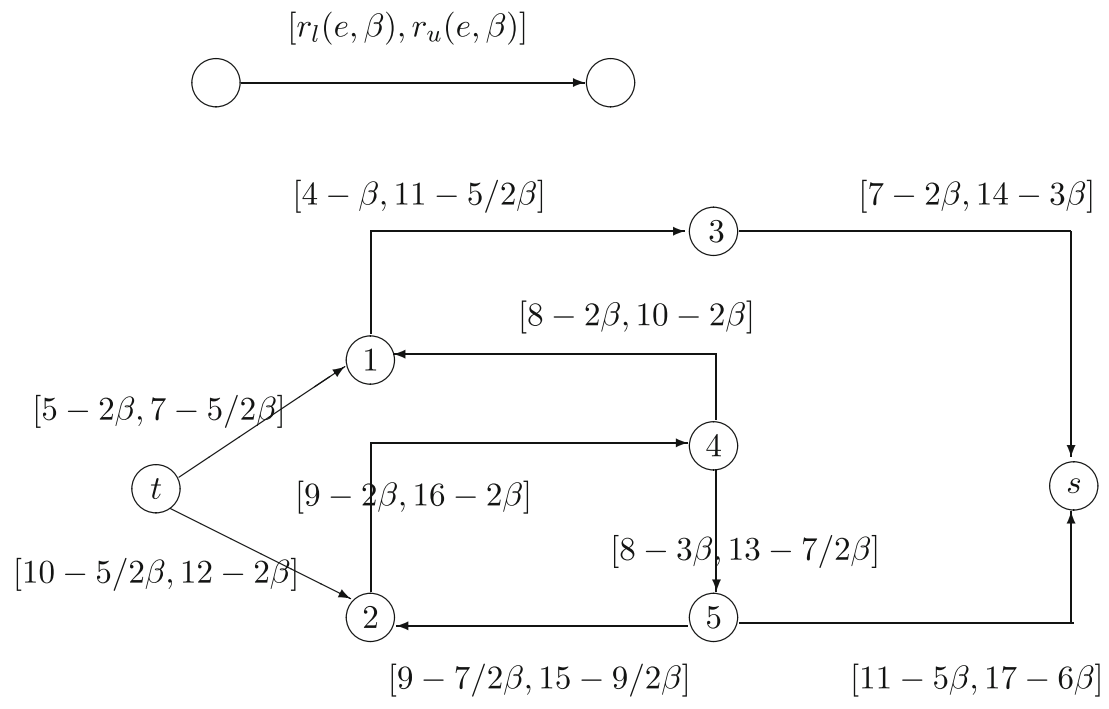

Figure 3. The network corresponding to the $r-\beta$-interval min flow problem. 
Example. For a given $\beta$, figure 1 presents an example network with fuzzy bounds and flows. The minimum flow problem is solved using $l-\beta$-interval and $r-\beta$-interval min flow problems on networks presented on figures 2 and 3.

\section{Conclusion}

Ghiyasvand (2011) presented a new method to solve the minimum cost flow problem with interval data, which solves the problem using two minimum cost flow problems with crisp data. This paper extended the method of Ghiyasvand (2011) to solve the minimum flow problem with interval lower and upper bounds, and flows using two minimum flow problems with crisp data. Then, this method is used to solve the minimum flow problem with fuzzy lower and upper bounds, and flows.

\section{References}

Bang-Jenson J and Gutin G 2001 Digraphs: Theory, algorithms and applications. London: Springer-Verlag Bondia J, Sala A, Pico J and Sainz M A 2006 Controller design under fuzzy pole-placement specifications: an interval arithmetic approach. IEEE T. Fuzzy Systems 14: 822-836

Ciupala L 2006 A deficit scaling algorithm for the minimum flow problem. Sadhana 31: 1169-1174

Ciupala L and Ciurea E 2001a Algorithms for minimum flows. Computer Scince Journal of Moldova 9: 275-290

Ciupala L and Ciurea E 2001b An approach of the minimum flow problem. The Fith International Symposium of Economic Informatics, 786-790

Ciupala L and Ciurea E 2003 An algorithm for the minimum flow problem. The Sixth International Conference of Economic Informations, 167-170

Ciupala L and Ciurea E 2004 Sequential and parallel algorithms for minimum flows. J. Appl. Mathemat. and Comput. 15: 53-78

Ciupala L and Ciurea E 2007 A highest-label preflow algorithm for the minimum flow problem, Proceedings of 11th WSEAS International Conference on Computers, 26-28

Ciupala L and Ciurea E 2008 About preflow algorithms for the minimum flow problem. WSEAS Transactions on Computer Research 3: 35-41

Ciurea E 2008 The wave preflow algorithm for the minimum flow problem, Proceeding of the 10th WSEAS International Conference on Mathematical and Computational Methods in Science and Engineering, 473-476

Ciurea E and Deaconu A 2007 Inverse minimum flow problem, J. Appl. Mathemat. and Comput. 23: $193-203$

Ciurea E and Deaconu A 2009 Minimum flows in bipartite networks with unit capacities, Proceedings of the 13th WSEAS International Conference on Computers, 313-317

Ciurea E, Georgescu O and Iolu M 2008a Minimum flow algorithms. Dynamic tree implementations. Studia University Babes-Bolyal Information 1: 73-82

Ciurea E, Georgescu O and Marinescu D 2008b Improved algorithms for minimum flows in bipartite networks. Int. J. Comput. 4: 351-360

Diamond P 2001 A fuzzy max-flow min-cut theorem. Fuzzy Sets and Systems 119: 139-148

Ghiyasvand M 2011 A new approach for solving the minimum cost flow problem with interval and fuzzy data. International Journal of Uncertainty Fuzziness and Knowledege-Based Systems 19: 71-88

Hanss M 2005 Applied fuzzy arithmetic: An introduction with engineering applications. Berlin, Springer 
Moore R E 1978 Methods and applications of interval analysis. Philadelphia: SIAM

Nguyen H T 1978 A note on the extension principle for fuzzy sets. J. Math. Anal. and Appl. 64: 359-380

Nguyen H T and Kreinovich V 1996 Nested intervals and sets: Concepts, relations to fuzzy sets, and applications. In: R B Kearfott and V Kreinovich (eds) Applications of Interval Computations. Dordrecht: Kluwer, 245-290 